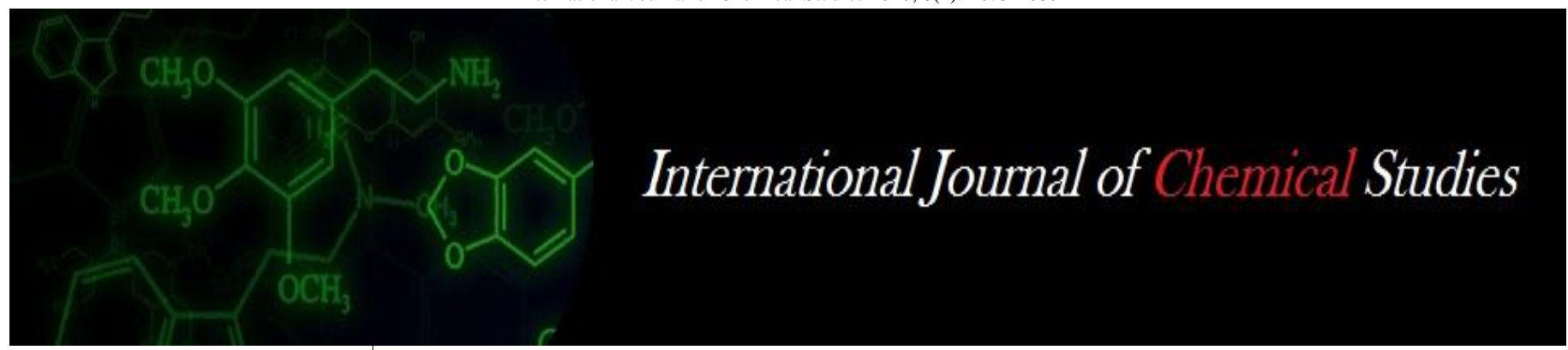

P-ISSN: 2349-8528

E-ISSN: 2321-4902

www.chemijournal.com

IJCS 2020; 8(4): 1673-1680

(C) 2020 IJCS

Received: 07-05-2020

Accepted: 09-06-2020

\section{Sudha Rani}

Scientist (Entomology),

Agricultural Research Station,

Vuyyuru, Krishna District,

Andhra Pradesh, India

P Mohana Rao

Scientist (Soil Science),

Agricultural Research Station,

Vuyyuru, Krishna District,

Andhra Pradesh, India

\section{K Krishnamma}

Principal Scientist

(Pl. Pathology), Agricultura

Research Station, Vuyyuru,

Krishna District,

Andhra Pradesh, India

\section{Efficacy of chlorantraniliprole $625 \mathrm{~g} / \mathrm{L}$ FS (Lumivia) for the management of stem borer and leaf folder in direct seeded rice}

\author{
D Sudha Rani, P Mohana Rao and K Krishnamma
}

DOI: $\underline{\text { https://doi.org/10.22271/chemi.2020.v8.i4q.9851 }}$

\begin{abstract}
The major production constraint of rice in enhancing productivity is due to attack by pests. Rice stem borer and leaf folder are considered as economic pests and influence the yield if proper timely management practices are not adopted. In case of severe pest incidences the usage of insecticides is inevitable. Among various modes of application of insecticides, seed treatment is mostly advantageous as it is required in small amount and offers control of target pests and ensuing the establishment of healthy and vigorous plants. Hence, studies on efficacy pertaining to Chlorantraniliprole $625 \mathrm{~g} / \mathrm{L}$ FS (lumivia) at different concentrations along with check chemicals viz., cartap hydrochloride 4G and untreated control were evaluate at Agricultural Research Station, Vuyyuru during kharif, 2019 and rabi 2019-2020 to assess the efficacy of various test chemicals against stem borer and leaf folder infesting direct sown rice. The experimental trial was laid out with seven treatments that were replicated four times and treatments were imposed as seed dresser and broadcasted as direct sown rice. The data on per dead hearts and per cent leaf folder damaged leaves at 30, 40,50,60 and 70 days after sowing were recorded for both rice variety (MTU 1061) and rice hybrid (28P67). The results revealed that, among various test chemicals the treatment $\mathrm{T}_{4}$ (Chlorantraniliprole 625g/L FS @ $90 \mathrm{~g}$ a.i./ha) had registered highest per cent reduction of stem borer with $76.71 \& 68.60$ in kharif and rabi seasons of study period for rice variety. With respect to rice hybrid the per cent reduction of stem borer incidence over control was $78.19 \& 65.09$ per cent in kharif and rabi, 2019-2020, respectively. Similarly, the leaf folder damaged leaves were also recorded least in $T_{4}$ with $61.06 \& 62.96$ per cent reduction over control with respect to rice variety during kharif and rabi, 2019-2020, respectively, whereas the corresponding values for rice hybrid was 61.04 and 71.64 during kharif and rabi seasons, respectively. Hence, it can be concluded that chlorantraniliprole $625 \mathrm{~g} / \mathrm{L}$ FS @90 g a.i./ha@ (lumivia) is effective as seed dresser in suppressing stem borer and leaf folder in direct seeded rice with no phytotoxicity on rice crop and no adverse effects on natural enemies.
\end{abstract}

Keywords: Chlorantraniliprole, management, stem borer, direct seeded rice

\section{Introduction}

Rice, being the major crop constitutes about 52 per cent of the total food grain production and 55 per cent of total cereal production in our country (Kakde and Patel, 2014) ${ }^{[1]}$. In India, rice is grown in 43.86 million ha, the production level is 104.80 million tones and the productivity is about $2390 \mathrm{~kg} \mathrm{ha}^{-1}$. In Andhra Pradesh it is cultivated in an area of 38.09 lakh ha with a production of 127.24 lakh tons and $4234 \mathrm{~kg} \mathrm{ha}^{-1}$ productivity (www. India stat.com) ${ }^{2}$. Insectpests are the major constraints in enhancing the rice productivity, besides diseases and ${ }^{2}$ weeds (Behura et al. 2011) ${ }^{[3]}$.

Approximately 21 per cent of the global production losses of rice are attributed to the attack of insect pests (Yarasi et al., 2008) ${ }^{[4]}$. Globally, stem borer alone causes yield losses of 10 million tones and accounts 50 per cent of all insecticides usage in the rice field (Deka and Barthakur, 2010) ${ }^{[5]}$. Among stem borers, the yellow stem borer (YSB), Scirpophaga incertulas (Walker) (Lepidoptera: Pyralidae) is the dominant species in India and rice plants are most prone to stem borer infestation at the tillering and flowering stages. Stem borer inflicted 18 to $40 \%$ damage to the rice crop. The rice leaf folder, Cnaphalocrocis medinalis (Guenee) earlier considered as a minor pest has gained the status of major pest with the widespread of high-yielding rice varieties and the accompanying changes in cultural practices (Teng et al. 1993) ${ }^{[6]}$. It was most widely spread and damage caused from 18.30 to 58.40 per

\section{Sudha Rani}

Scientist (Entomology), Agricultural Research Station,

Vuyyuru, Krishna district,

Andhra Pradesh, India 
cent depending upon the stage of the crop at the time of infestation (Ramasamy and Jaliecksono 1996) ${ }^{[7]}$. Because of cultivating high yielding varieties year after year which are susceptible to pest attack insecticides remain as sole dependable weapon to the farmer in order to mitigate the insect population especially during epidemics. But among various modes of use of insecticides, seed treatment was the most appreciable method as for seed treatment only small amounts of pesticides are required compared to broadcast sprays which ensure no environmental risks and human hazards. Hence, a field trial was conducted at Agricultural Research Station, Vuyyuru, Andhra Pradesh, India during kharif, 2019 and rabi, 2019-2020 to evaluate the bioefficacy of Chlorantraniliprole 625g/L FS (Lumivia) as seed dresser in direct sown rice in suppressing the major pests incidence i.e., stem borer and leaf folder.

\section{Material and Methods}

\subsection{Experimental site}

Field studies were carried out at Agricultural Research Station, Vuyyuru, Krishna district, Andhra Pradesh during kharif, 2019 and rabi, 2019-2020 to evaluate the efficacy of test chemical Chlorantraniliprole $625 \mathrm{~g} / \mathrm{L}$ FS (Lumivia) at different concentrations in comparison to cartap hydrochloride. The experiment was laid out in RBD design with a plot size of $5 \times 5 \mathrm{~m}$ including seven treatments which were replicated four times. Judicious fertilizer, inter cultivation and other agronomic practices were employed as per recommendations. Rice variety MTU 1061 and rice hybrid 28P67 which were prone to pest attack was used for experimentation. Direct sown (broadcasting) method was employed for cultivation with seed rate of 30 and $15 \mathrm{~kg}$ per hectare for rice variety and rice hybrid, respectively.

\subsection{Imposition of treatments}

To evaluate efficacy of Chlorantraniliprole 625g/L FS (lumivia), a total of seven treatments including one test chemical cartap hydrochloride $4 \mathrm{G}$ and untreated control were imposed as seed treatment. The details of imposed treatments for rice variety and rice hybrid were presented in Table 1 . The experimentation was carried out with rice variety and rice hybrid with same treatments simultaneously.

Table 1: Seed treatment details for pest management in direct sown rice

\begin{tabular}{|c|c|c|c|c|c|c|c|}
\hline \multirow{2}{*}{ Treatment No } & & Treatment & \multirow{2}{*}{$\begin{array}{c}\text { Dose } \\
(\text { g.i./ha }\end{array}$} & \multicolumn{2}{c|}{ Dose $(\mathbf{g}$ a.i./kg seed) } & \multicolumn{2}{c|}{ Formulation (ml/kg seed) } \\
\hline & & & Variety & Hybrid & Variety & Hybrid \\
\hline $\mathrm{T}_{1}$ & $:$ & chlorantraniliprole 625g/L FS & 67.5 & 2.25 & 4.5 & 3.6 & 7.2 \\
\hline $\mathrm{T}_{2}$ & $:$ & chlorantraniliprole 625g/L FS & 75.0 & 02.5 & 5.0 & 4.0 & 8.0 \\
\hline $\mathrm{T}_{3}$ & $:$ & chlorantraniliprole 625g/L FS & 82.5 & 2.75 & 5.5 & 4.4 & 8.8 \\
\hline $\mathrm{T}_{4}$ & $:$ & chlorantraniliprole 625g/L FS & 90.0 & 3.00 & 6.0 & 4.8 & 9.6 \\
\hline $\mathrm{T}_{5}$ & $:$ & chlorantraniliprole 625g/L FS & 180.0 & 6.00 & 12.0 & 9.6 & 19.2 \\
\hline $\mathrm{T}_{6}$ & $:$ & cartap hydrochloride 4\% G & 750.0 & - & - & $18750 \mathrm{~g}$ & $18750 \mathrm{~g}$ \\
\hline $\mathrm{T}_{7}$ & $:$ & untreated control (water) & - & - & - & - & - \\
\hline
\end{tabular}

2.2.1 Methodology: Required quantity of chlorantraniliprole $625 \mathrm{~g} / \mathrm{L}$ FS as per given dosage were measured and mixed with adequate water to make slurry sufficient to treat the seeds. Applied the slurry onto seeds in a plastic bag and shaken to coat seeds uniformly with insecticide slurry. Treated seeds were dried in shade prior to sowing.

\subsection{Meteorological data}

The data on weather parameters viz., maximum temperature, minimum temperature, morning and evening relative humidity and rainfall data was recorded from Meteorological unit installed at Agricultural Research Station, Vuyyuru.

\subsubsection{Data recording on pest incidence}

The data on stem borer incidence in terms of per cent Dead Hearts (DH) was recorded from 20 hills per each plot at 30, 40, 50, 60 and 70 days after sowing.

$\mathrm{DH}$ per cent $=\frac{\text { Total number of dead hearts in } 20 \text { hills }}{\text { Total number of tillers in } 20 \text { hills }} \times 100$

Similarly, per cent number of leaf folder larvae per hill was observed per plot at 30, 40, 50, 60 and 70 days after sowing. The leaf folder damage was estimated using a rate scale (0-5) from randomly selected hills (Table 2). The leaf folder damage in leaves was calculated as per formulae hereunder at 30, 40, 50, 60 and 70 days after sowing.

\footnotetext{
Per cent damaged leaves $=\frac{\text { Total number of damaged leaves in } 20 \text { hills }}{\text { Total }}$
} $\mathrm{X} 100$
The per cent reduction of stem borer and leaf folder damage in various treatments over control was calculated as per formulae hereunder.

\section{Per cent reduction over control $=\frac{\mathrm{C}-\mathrm{T}}{\mathrm{C}} \times 100$}

Where $\mathrm{C}=$ pest incidence in control; $\mathrm{T}=$ pest incidence in treatment

Table 2: leaf damage scale for rice leaf folder

\begin{tabular}{|c|c|}
\hline Scale & parameter \\
\hline 1 & No damage/ scrapping on leaves/hill \\
\hline 2 & $10-30 \%$ damage on leaves/hill \\
\hline 3 & $30-50 \%$ damage on leaves/hill \\
\hline 4 & $50-80 \%$ damage on leaves/hill \\
\hline 5 & $>80 \%$ damage on leaves/hill \\
\hline
\end{tabular}

\subsection{Influence on Natural enemies}

The effect of various treatments (test chemicals) on natural enemies (spiders and mirid bugs) was recorded from 20 hills at 30, 50 ad 70 days after sowing was recorded.

\subsection{Phytotoxicity}

The crop response or injury for yellowing, stunting, necrosis, epinasty, hyponasty were recorded in all the treatments and phytotoxicity rating was recorded basing on phytotoxicity scale (Table 3). 
Table 3: Phytotoxicity scale based on crop injury

\begin{tabular}{|c|c|c|c|}
\hline Scale & Crop injury (\%) & Scale & Crop injury (\%) \\
\hline 0 & 0 & 6 & $51-60$ \\
\hline 1 & $1-10$ & 7 & $61-70$ \\
\hline 2 & $11-20$ & 8 & $71-80$ \\
\hline 3 & $21-30$ & 9 & $81-90$ \\
\hline 4 & $31-40$ & 10 & $91-100$ \\
\hline 5 & $41-50$ & & \\
\hline
\end{tabular}

\subsection{Yield data}

Plot-wise yields were also recorded after removing the two border rows from each plot and expressed in $\mathrm{Kg} \mathrm{ha}^{-1}$ and accordingly the yield advantage over control was retrieved.

\subsection{Data analysis}

The recorded data was transformed with suitable transformation method before analysis and subjected to analysis of variance. Significant differences in means were separated using Duncan's multiple range test $(\mathrm{P}=0.05)$.

\section{Results and discussions \\ 3.1. Meteorological data}

During the crop season kharif, 2019 the average maximum $\left(33.6{ }^{\circ} \mathrm{C}\right)$ and minimum temperature $\left(17.40{ }^{\circ} \mathrm{C}\right)$ was recorded during August and December, 2019 respectively. During rabi, 2019-2020 the maximum temperature was recorded in April $2020\left(38.14{ }^{\circ} \mathrm{C}\right)$ and minimum temperature was recorded in January $2020\left(15.52^{\circ} \mathrm{C}\right)$. The highest rainfall was recorded in month September for kharif, 2019 and February for rabi, 2019-2020 with 228.3 and $13.21 \mathrm{~mm}$, respectively (Table 4).

Table 4: Meteorological Data, Agricultural Research Station, Vuyyuru 2019-2020

\begin{tabular}{|c|c|c|c|c|c|}
\hline Month and year & \multicolumn{2}{|c|}{ Temperature $\left(\mathbf{(}^{\mathbf{c})}\right.$} & Humidity & Rainfall & Sunshine hrs \\
\hline & Max & Min & $(\mathbf{m m})$ & (mm & \\
\hline August 2019 & 33.68 & 21.14 & 60.45 & 146.5 & 6.2 \\
\hline September 2019 & 33.73 & 23.38 & 63.15 & 228.3 & 5.7 \\
\hline October 2019 & 32.72 & 22.88 & 63.94 & 43.4 & 6.8 \\
\hline November 2019 & 31.07 & 19.77 & 61.25 & 0.00 & 6.2 \\
\hline December 2019 & 29.61 & 17.14 & 55.14 & 0.00 & 7.2 \\
\hline January 2020 & 30.71 & 15.52 & 64.92 & 2.00 & 7.1 \\
\hline February 2020 & 33.24 & 16.37 & 51.78 & 13.21 & 7.1 \\
\hline March 2020 & 36.36 & 18.60 & 64.88 & 0.00 & 7.5 \\
\hline April 2020 & 38.14 & 23.41 & 51.27 & 5.10 & 8.9 \\
\hline
\end{tabular}

\subsection{Efficacy of chlorantraniliprole $625 \mathrm{~g} / \mathrm{L} \mathrm{FS}$ on incidence} of major pests of rice during kharif, 2019

The efficacy of the test chemical, chlorantraniliprole $625 \mathrm{~g} / \mathrm{L}$ FS as seed dresser on incidence of rice stem borer in terms of per cent dead hearts and incidence of leaf folder in terms of larval population and damaged leaves was recorded at 30, 40, 50,60 and 70 days after sowing (DAS) in both rice variety and rice hybrid.

\subsubsection{Influence of test chemical on incidence of rice stem borer}

The incidence of rice stem borer in terms of dead hearts had ranged from 3.3 to 25.5 per cent in rice variety and 4.5 to 29.5 per cent in rice hybrid during kharif, 2019.
3.2.1.2 Rice variety: In rice variety MTU 1061 among various treatments, $\mathrm{T}_{4}$ (chlorantraniliprole $625 \mathrm{~g} / \mathrm{L} \mathrm{FS} @ 90 \mathrm{~g}$ a.i. /ha) had registered lowest stem borer incidence with only 5.4, 3.3, 3.8, 5.3 and 7.8 per cent dead hearts as against highest incidence in untreated control recording 18.4, 20.5, 21.5, 23.4 and 25.5 per cent at 30, 40, 50, 60 and 70 DAS, respectively. The next better treatment in suppressing per cent dead heart was found to be $\mathrm{T}_{6}$ (cartap hydrochloride 4\% G @ $750 \mathrm{~g}$ a.i./ha) with $7.5,6.5,6.5,8.4$ and 16.8 per cent dead hearts at 30,40,50, 60 and 70 DAS, respectively. The other treatments also exhibited better efficacy compared to untreated control and the chronological order in suppressing the borer incidence represents $\mathrm{T}_{4}>\mathrm{T}_{6}>\mathrm{T}_{2}>\mathrm{T}_{3}>\mathrm{T}_{1}>\mathrm{T}_{7}$ with 5.1, 9.1, 11.8, 12.1, 14.1 and 21.9 per cent, respectively (Table 5).

Table 5: Efficacy of chlorantraniliprole 625g/L FS against rice stem borer in terms of per cent dead hearts during kharif, 2019

\begin{tabular}{|c|c|c|c|c|c|c|c|c|c|c|c|c|}
\hline & \multicolumn{5}{|c|}{ Rice variety } & \multicolumn{7}{|c|}{ Rice Hybrid } \\
\hline Treatments & DAS & 40 DAS & 50 DAS & 60 DAS & 70 DAS & & 30 DAS & 40 DAS & 50 DAS & 60 DAS & 70 DAS & \\
\hline $\mathrm{T}_{1}$ & $\begin{array}{c}11.8 \\
(20.09)^{\mathrm{c}}\end{array}$ & $\begin{array}{c}12.5 \\
(20.70)^{\mathrm{c}}\end{array}$ & $\begin{array}{c}12.2 \\
(20.44)^{\mathrm{c}}\end{array}$ & $\begin{array}{c}15.5 \\
(23.18)^{b}\end{array}$ & $\begin{array}{c}18.5 \\
(25.47)^{\mathrm{b}}\end{array}$ & $\begin{array}{c}14.1 \\
(22.06)^{b}\end{array}$ & $\begin{array}{c}15.8 \\
(23.42)^{\mathrm{c}}\end{array}$ & $\begin{array}{c}13.8 \\
(21.81)^{\mathrm{c}}\end{array}$ & $\begin{array}{c}12.7 \\
(20.88)^{\mathrm{b}}\end{array}$ & $\begin{array}{c}14.6 \\
(22.46)^{\mathrm{b}}\end{array}$ & $\begin{array}{c}19.8 \\
(26.42)^{\mathrm{b}}\end{array}$ & $\begin{array}{c}15.3 \\
(23.03)^{b}\end{array}$ \\
\hline $\mathrm{T}_{2}$ & 10.5 & $\begin{array}{c}9.3 \\
(17.76)^{\mathrm{bc}}\end{array}$ & $=\begin{array}{c}8.5 \\
(16.95)^{b}\end{array}$ & $\begin{array}{c}13.3 \\
(21.39)^{\mathrm{b}}\end{array}$ & $\begin{array}{c}17.5 \\
(24.73)^{\mathrm{b}}\end{array}$ & $\begin{array}{c}11.8 \\
(20.09)^{b}\end{array}$ & $\begin{array}{c}14.3 \\
(22.22)^{\mathrm{c}}\end{array}$ & $\begin{array}{c}10.5 \\
(18.91)^{\mathrm{bc}}\end{array}$ & $\begin{array}{c}9.8 \\
(18.24)^{b}\end{array}$ & $\begin{array}{c}10.8 \\
(19.19)^{\mathrm{ab}}\end{array}$ & $\begin{array}{c}20.5 \\
(26.92)^{\mathrm{b}}\end{array}$ & $\begin{array}{c}13.2 \\
(21.30)^{\mathrm{b}}\end{array}$ \\
\hline $\mathrm{T}_{3}$ & & $\begin{array}{c}7.8 \\
(16.22)^{\mathrm{b}}\end{array}$ & $\begin{array}{c}8.0 \\
(16.43)^{b}\end{array}$ & $\begin{array}{c}15.1 \\
(22.87)^{\mathrm{b}}\end{array}$ & $\begin{array}{c}19.5 \\
(26.21)^{b}\end{array}$ & $\begin{array}{c}12.1 \\
(20.36)^{b}\end{array}$ & $\begin{array}{r}11 \\
(19.8\end{array}$ & $\begin{array}{c}9.8 \\
(18.24)^{b}\end{array}$ & $\begin{array}{r}8 . \\
(17.1\end{array}$ & $\begin{array}{c}11.3 \\
(19.64)^{\mathrm{ab}}\end{array}$ & $\begin{array}{c}19 \\
(25 .\end{array}$ & $\begin{array}{c}12.1 \\
(20.36)^{\mathrm{b}}\end{array}$ \\
\hline $\mathrm{T}_{4}$ & $\begin{array}{c}5.4 \\
(13.44)^{\mathrm{a}}\end{array}$ & $\begin{array}{c}3.3 \\
(10.47)^{\mathrm{a}}\end{array}$ & $\begin{array}{r}3.8 \\
(11.24\end{array}$ & $\begin{array}{c}5.3 \\
(13.31)^{\mathrm{a}}\end{array}$ & $\begin{array}{c}7.8 \\
(16.22)^{\mathrm{a}}\end{array}$ & $\begin{array}{c}5.1 \\
(13.05)^{\mathrm{a}}\end{array}$ & $\begin{array}{r}5 . \\
(13.3\end{array}$ & $\begin{array}{c}5.0 \\
(12.92)^{\mathrm{a}}\end{array}$ & $\begin{array}{r}4 . \\
(12 .\end{array}$ & $\begin{array}{c}5.8 \\
(13.94)^{\mathrm{a}}\end{array}$ & $\begin{array}{r}8 . \\
(16 .\end{array}$ & $\begin{array}{c}5.8 \\
(13.94)^{\mathrm{a}}\end{array}$ \\
\hline $\mathrm{T}_{6}$ & $\begin{array}{c}7.5 \\
(15.89)^{\mathrm{ab}}\end{array}$ & $\begin{array}{c}6.5 \\
(14.77)^{\mathrm{b}}\end{array}$ & $\begin{array}{c}6.5 \\
(14.77)^{b}\end{array}$ & $\begin{array}{c}8.4 \\
(16.85)^{\mathrm{a}} \\
\end{array}$ & $\begin{array}{c}16.8 \\
(24.20)^{\mathrm{b}}\end{array}$ & \begin{tabular}{|c|}
9.1 \\
$(17.56)^{b}$ \\
\end{tabular} & $\begin{array}{c}6.8 \\
(15.12)^{\mathrm{a}} \\
\end{array}$ & $\begin{array}{c}5.4 \\
(13.44)^{\mathrm{a}} \\
\end{array}$ & $\begin{array}{c}5.1 \\
(13.05)^{\mathrm{a}}\end{array}$ & $\begin{array}{c}7.1 \\
(15.45)^{\mathrm{a}}\end{array}$ & $\begin{array}{c}10.4 \\
(18.81)^{\mathrm{a}} \\
\end{array}$ & $\begin{array}{c}7.0 \\
(15.34)^{\mathrm{a}}\end{array}$ \\
\hline $\mathrm{T}_{7}$ & $\begin{array}{c}18.4 \\
(25.40)^{\mathrm{d}}\end{array}$ & $\begin{array}{c}20.5 \\
(26.92)^{d}\end{array}$ & $\begin{array}{c}21.5 \\
(27.62)^{d}\end{array}$ & $\begin{array}{c}23.4 \\
(28.93)^{\mathrm{b}}\end{array}$ & $\begin{array}{c}25.5 \\
(30.33)^{\mathrm{c}}\end{array}$ & $\begin{array}{c}21.9 \\
(27.90)^{\mathrm{c}}\end{array}$ & $\begin{array}{c}23.2 \\
(28.79)^{\mathrm{d}}\end{array}$ & $\begin{array}{c}25.8 \\
(30.53)^{\mathrm{c}}\end{array}$ & $\begin{array}{c}26.5 \\
(30.98)^{\mathrm{c}}\end{array}$ & $\begin{array}{c}28.0 \\
(31.95)^{\mathrm{c}}\end{array}$ & $\begin{array}{c}29.5 \\
(32.90)^{\mathrm{c}}\end{array}$ & $\begin{array}{c}26.6 \\
(31.05)^{\mathrm{c}}\end{array}$ \\
\hline & & & & 4.01 & & & & 3. & & 4 & & 2.68 \\
\hline $\mathrm{CV}$ & 18.5 & 12.4 & 13.9 & 17.6 & 9.55 & 13.54 & 16.55 & 12.54 & 10.57 & 14.55 & 10.54 & 10.15 \\
\hline
\end{tabular}

Mean with same letter are not significantly different at 5\% level by Duncan's Multiple Range test

Values in parenthesis are arc sine transformed values; DAS: Days After Sowin 
3.2.1.3 Rice hybrid: In rice hybrid $28 \mathrm{P} 67$ also similar trend was observed with respect to efficacy of various treatments in reducing stemborer incidence as in case with rice variety. $\mathrm{T}_{4}$ (chlorantraniliprole $625 \mathrm{~g} / \mathrm{L} \mathrm{FS} @ 90 \mathrm{~g}$ a.i. /ha) had recorded significantly minimum per cent dead hearts compared to all other treatments and found on par to $\mathrm{T}_{6}$ (cartap hydrochloride 4\% G @750 g a.i./ha). The per cent incidence of stem borer in $\mathrm{T}_{4}$ and $\mathrm{T}_{6}$ were recorded as $5.3 \& 6.8,5.0 \& 5.4,4.5 \& 5.1$, $5.8 \& 7.1$ and $8.5 \& 10.4$ per cent dead hearts at $30,40,50,60$ and 70 DAS, respectively and considered as afforded with minimum borer incidence. The highest per cent dead hearts were recorded in untreated control with 23.2, 25.8, 26.5, 28.0 and 29.5 at 30, 40, 50, 60 and 70 DAS, respectively. The increasing order in efficacy (average per cent dead hearts) of various treatments represents $\mathrm{T}_{4}(5.8)>\mathrm{T}_{6}(7.0)>\mathrm{T}_{2}(13.2)>\mathrm{T}_{3}$ (12.1) $>\mathrm{T}_{1}(15.3)>\mathrm{T}_{7}(26.6)$ (Table 5).

\subsubsection{Influence of test chemical on incidence of rice leaf folder in terms of larval population}

The leaf folder infestation in terms of larval population per hill was recorded and the results inferred that all the test molecules exerted better suppression of the pest incidence over control. The larval population of leaf folder ranged from
0.0 to 7.5 and 0.8 to 7.2 number per hill in rice variety and rice hybrid, respectively. More or less all the treatments found on par to each other except $\mathrm{T}_{1}$ (chlorantraniliprole $625 \mathrm{~g} / \mathrm{L} \mathrm{FS}$ @ $67.5 \mathrm{~g}$ a.i./ha) and $\mathrm{T}_{7}$ (untreated control).

The average leaf folder population per hill was found to be less in $\mathrm{T}_{4}$ ad $\mathrm{T}_{6}$ in both rice hybrid and rice variety with 2.3 and 1.5, respectively. The chronological order in suppressing leaf folder population was $\mathrm{T}_{4}(1.5 \& 2.3)>\mathrm{T}_{6}(2.1 \& 3.5)>\mathrm{T}_{3}$ $(2.5 \& 4.6)>\mathrm{T}_{2}(3.3 \& 5.0)>\mathrm{T}_{1}(4.1 \& 5.5)>\mathrm{T}_{7}(6.1 \& 6.6)$ (Table 6).

\subsubsection{Influence of test chemical on incidence of rice leaf folder in terms of per cent damage}

The leaf folder damage also followed the analogous trend as in case with larval population. At 30 DAS the leaf folder damage in rice variety found to be below ETL in all treatments except control. At 40, 50, 60 and 70 DAS, the per cent leaf folder damaged leaves were recorded to be less in $\mathrm{T}_{4}$ followed by $\mathrm{T}_{6}$ confirming them as the best treatments with $4.3 \& 5.3,3.5 \& 6.9,8.5 \& 9.3$ and $5.8 \& 6.9$ per cent in rice variety, respectively. The corresponding values for $\mathrm{T}_{4}$ and $\mathrm{T}_{6}$ with respect to rice hybrid were $5.3 \& 6.8,6.8 \& 7.9,8.9 \&$ 10.1 and $10.8 \& 12.5$ per cent respectively.

Table 6: Efficacy of chlorantraniliprole 625g/L FS on larval population of rice leaf folder/hill during kharif, 2019

\begin{tabular}{|c|c|c|c|c|c|c|c|c|c|c|c|c|}
\hline & \multicolumn{5}{|c|}{ Rice variety } & \multicolumn{7}{|c|}{ Rice Hybrid } \\
\hline Treatments & 30 DAS & 40 DAS & 50 DAS & 60 DAS & 70 DAS & Average & 30 DAS & 40 DAS & 50 DAS & 60 DAS & 70 DAS & Average \\
\hline $\mathrm{T}_{1}$ & $\begin{array}{c}1.5 \\
(1.22)^{\mathrm{a}}\end{array}$ & $\begin{array}{c}3.4 \\
(1.84)^{\mathrm{bc}}\end{array}$ & $\begin{array}{c}5.3 \\
(2.30)^{\mathrm{b}}\end{array}$ & $\begin{array}{c}4.8 \\
(2.19)^{\mathrm{bc}}\end{array}$ & $\begin{array}{c}5.7 \\
(2.39)^{\mathrm{bc}}\end{array}$ & $\begin{array}{c}4.1 \\
(2.02)^{\mathrm{b}}\end{array}$ & $\begin{array}{c}3.8 \\
(1.95)^{c}\end{array}$ & $\begin{array}{c}6.3 \\
(2.51)^{\mathrm{cd}}\end{array}$ & $\begin{array}{c}6.9 \\
(2.63)^{\mathrm{d}}\end{array}$ & $\begin{array}{c}4.3 \\
(2.07)^{\mathrm{b}}\end{array}$ & $\begin{array}{c}6.3 \\
(2.51)^{\mathrm{b}}\end{array}$ & $\begin{array}{c}5.5 \\
(2.35)^{c}\end{array}$ \\
\hline $\mathrm{T}_{2}$ & $\begin{array}{c}0.9 \\
(0.95)^{\mathrm{a}}\end{array}$ & $\begin{array}{c}2.8 \\
(1.67)^{\mathrm{bc}}\end{array}$ & $\begin{array}{c}4.8 \\
(2.19)^{\mathrm{b}}\end{array}$ & $\begin{array}{c}3.7 \\
(1.92)^{\mathrm{ab}}\end{array}$ & $\begin{array}{c}4.3 \\
(2.07)^{\mathrm{ab}}\end{array}$ & $\begin{array}{c}3.3 \\
(1.82)^{\mathrm{a}}\end{array}$ & $\begin{array}{c}3.5 \\
(1.87)^{c}\end{array}$ & $\begin{array}{c}5.4 \\
(2.32)^{\mathrm{bc}}\end{array}$ & $\begin{array}{c}6.0 \\
(2.45)^{\mathrm{c}}\end{array}$ & $\begin{array}{c}4.0 \\
(2.00)^{\mathrm{b}}\end{array}$ & $\begin{array}{c}6.0 \\
(2.45)^{b}\end{array}$ & $\begin{array}{c}5.0 \\
(2.24)^{\mathrm{b}}\end{array}$ \\
\hline $\mathrm{T}_{3}$ & $\begin{array}{c}0 \\
(0.0)^{\mathrm{a}}\end{array}$ & $\begin{array}{c}1.3 \\
(1.14)^{\mathrm{ab}}\end{array}$ & $\begin{array}{c}3.5 \\
(1.87)^{\mathrm{ab}}\end{array}$ & $\begin{array}{c}3.5 \\
(1.87)^{\mathrm{ab}}\end{array}$ & $\begin{array}{c}4.0 \\
(2.00)^{\mathrm{a}}\end{array}$ & $\begin{array}{c}2.5 \\
(1.58)^{\mathrm{a}}\end{array}$ & $\begin{array}{c}3.0 \\
(1.73)^{\mathrm{bc}}\end{array}$ & $\begin{array}{c}5.3 \\
(2.30)^{\mathrm{b}}\end{array}$ & $\begin{array}{c}5.4 \\
(2.32)^{\mathrm{bc}}\end{array}$ & $\begin{array}{c}3.6 \\
(1.90)^{b}\end{array}$ & $\begin{array}{c}5.5 \\
(2.35)^{\mathrm{ab}}\end{array}$ & $\begin{array}{c}4.6 \\
(2.14)^{\mathrm{b}}\end{array}$ \\
\hline $\mathrm{T}_{4}$ & $\begin{array}{c}0 \\
(0.0)^{\mathrm{a}}\end{array}$ & $\begin{array}{c}0 \\
(0.0)^{\mathrm{a}}\end{array}$ & $\begin{array}{c}1.8 \\
(1.34)^{\mathrm{a}}\end{array}$ & $\begin{array}{c}2.2 \\
(1.48)^{\mathrm{a}}\end{array}$ & $\begin{array}{c}3.6 \\
(1.90)^{\mathrm{a}}\end{array}$ & $\begin{array}{c}1.5 \\
(1.22)^{\mathrm{a}}\end{array}$ & $\begin{array}{c}0.8 \\
(0.89)^{\mathrm{a}}\end{array}$ & $\begin{array}{c}3.6 \\
(1.90)^{\mathrm{a}}\end{array}$ & $\begin{array}{c}3.1 \\
(1.76)^{\mathrm{a}}\end{array}$ & $\begin{array}{c}2.2 \\
(1.48)^{\mathrm{a}}\end{array}$ & $\begin{array}{c}2.0 \\
(1.41)^{\mathrm{a}}\end{array}$ & $\begin{array}{c}2.3 \\
(1.52)^{\mathrm{a}}\end{array}$ \\
\hline $\mathrm{T}_{6}$ & $\begin{array}{c}0 \\
(0.0)^{\mathrm{a}}\end{array}$ & $\begin{array}{c}1.1 \\
(1.05)^{\mathrm{a}}\end{array}$ & $\begin{array}{c}2.9 \\
(1.70)^{\mathrm{a}}\end{array}$ & $\begin{array}{c}2.4 \\
(1.55)^{\mathrm{a}}\end{array}$ & $\begin{array}{c}4.2 \\
(2.05)^{\mathrm{ab}}\end{array}$ & $\begin{array}{c}2.1 \\
(1.45)^{\mathrm{a}}\end{array}$ & $\begin{array}{c}1.8 \\
(1.34)^{\mathrm{ab}}\end{array}$ & $\begin{array}{c}4.4 \\
(2.10)^{\mathrm{ab}}\end{array}$ & $\begin{array}{c}4.8 \\
(2.19)^{\mathrm{b}}\end{array}$ & $\begin{array}{c}2.9 \\
(1.70)^{\mathrm{ab}}\end{array}$ & $\begin{array}{c}3.4 \\
(1.84)^{\mathrm{a}}\end{array}$ & $\begin{array}{c}3.5 \\
(1.87)^{\mathrm{ab}}\end{array}$ \\
\hline $\mathrm{T}_{7}$ & $\begin{array}{c}5.5 \\
(2.35)^{b}\end{array}$ & $\begin{array}{c}5.4 \\
(2.32)^{\mathrm{c}}\end{array}$ & $\begin{array}{c}6.0 \\
(2.45)^{\mathrm{b}}\end{array}$ & $\begin{array}{c}6.3 \\
(2.51)^{c}\end{array}$ & $\begin{array}{c}7.5 \\
(2.74)^{c}\end{array}$ & $\begin{array}{c}6.1 \\
(2.47)^{c}\end{array}$ & $\begin{array}{c}4.3 \\
(2.07)^{c}\end{array}$ & $\begin{array}{c}7.8 \\
(2.79)^{\mathrm{d}}\end{array}$ & $\begin{array}{c}8.4 \\
(2.90)^{\mathrm{e}}\end{array}$ & $\begin{array}{c}5.3 \\
(2.30)^{c}\end{array}$ & $\begin{array}{c}7.2 \\
(2.68)^{\mathrm{b}}\end{array}$ & $\begin{array}{c}6.6 \\
(2.57)^{c}\end{array}$ \\
\hline $\mathrm{CD}$ & 1.38 & 1.15 & 0.69 & 0.57 & 0.27 & 0.81 & 0.44 & 0.32 & 0.18 & 0.29 & 1.04 & 0.45 \\
\hline $\mathrm{CV}$ & 13.5 & 19.8 & 11.4 & 10.4 & 15.8 & 14.18 & 10.5 & 13.2 & 10.5 & 13.4 & 18.4 & 10.8 \\
\hline
\end{tabular}

Mean with same letter are not significantly different at 5\% level by Duncan's Multiple Range test

Values in parenthesis are arc sine transformed values; DAS: Days After Sowing

The lowest average per cent damage leaves by leaf folder were registered with $\mathrm{T}_{4}(4.4 \& 6.7)$ followed by $\mathrm{T}_{6}(5.7 \&$ 8.0), $\mathrm{T}_{3}(7.5 \& 11.7), \mathrm{T}_{2}(8.7 \& 12.4), \mathrm{T}_{1}(10.1 \& 13.4)$ and $\mathrm{T}_{7}$ (11.3 \& 17.2) test chemicals in rice variety and rice hybrid during kharif 2019, respectively (Table 7).

\subsection{Efficacy of chlorantraniliprole $625 \mathrm{~g} / \mathrm{L}$ FS on incidence of major pests of rice during rabi, 2019-2020}

The efficacy of the test chemical, chlorantraniliprole $625 \mathrm{~g} / \mathrm{L}$ FS as seed dresser on incidence of rice stem borer in terms of per cent dead hearts and incidence of leaf folder in terms of larval population and damaged leaves was recorded at 30, 40, 50,60 and 70 days after sowing (DAS) in both rice variety and rice hybrid.

\subsubsection{Influence of test chemicals on incidence of rice stem borer}

The incidence of rice stem borer in terms of dead hearts had ranged from 1.8 to 10.5 and 2.8 to 13.8 per cent in rice variety and rice hybrid, respectively during rabi, 2019-2020.
3.3.1.1 Rice variety: In rice variety among various treatments, the minimum per cent incidence of stem borer was recorded in $\mathrm{T}_{4}$ (chlorantraniliprole $625 \mathrm{~g} / \mathrm{L} \mathrm{FS} @ 90 \mathrm{~g}$ a.i. /ha) treated plots with $2.2,1.8,3.2,4.3$ and 2.1 per cent dead hearts followed by $\mathrm{T}_{6}$ with $3.8,2.7,4.0,5.6$ and 3.8 per cent dead hearts as against highest per cent dead hearts in untreated control plots with $7.4,8.7,10.5,9.8$ and 6.8 per cent at 30, 40, 50, 60 and 70 DAS, respectively. The average per cent dead hearts during rabi, 2019-2020 was recorded less in $\mathrm{T}_{4}$ and $\mathrm{T}_{6}$ with 2.7 and 4.0 per cent dead hearts as against highest in untreated control $(8.6 \%)$. Next to $\mathrm{T}_{6}$ the plots treated with $\mathrm{T}_{3}(5.3 \%)$ followed by $\mathrm{T}_{2}(5.6 \%)$ and $\mathrm{T}_{1}(6.3 \%)$ had recorded less average per cent dead hearts compared to control.

3.3.1.2 Rice hybrid: In rice hybrid also the plots treated with $\mathrm{T}_{4}$ (chlorantraniliprole $625 \mathrm{~g} / \mathrm{L}$ FS @ $90 \mathrm{~g}$ a.i. /ha) had recorded significantly least per cent dead hearts with $3.2,2.8$, 3.8, 4.1 and 4.5 per cent at 30, 40, 50, 60 and 70 DAS, respectively. $\mathrm{T}_{4}$ treated plots found to be on par with per cent dead hearts damage in T6 treated plots with 4.3, 5.0, 4.8, 5.3 and 6.0 as against highest damage recorded in control $\left(\mathrm{T}_{7}\right)$ 
with $8.7,11.2,13.8,10.9 \& 8.5$ per cent at $30,40,50,60$ and 70 DAS, respectively. The average per cent dead hearts by stem borer during rabi, 2019-2020 was significantly lowest in
$\mathrm{T}_{4}$ (3.7) followed by $\mathrm{T}_{6}(5.1), \mathrm{T}_{3}(5.1), \mathrm{T}_{2}(5.7), \mathrm{T}_{1}(6.9)$ and $\mathrm{T}_{7}$ (10.6) (Table 8).

Table 7: Efficacy of chlorantraniliprole 625g/L FS against rice leaffolder in terms of per cent damage during kharif, 2019

\begin{tabular}{|c|c|c|c|c|c|c|c|c|c|c|c|c|}
\hline & \multicolumn{9}{|c|}{ Rice variety } & \multicolumn{9}{c|}{ Rice Hybrid } \\
\hline Treatments & 30 DAS & 40 DAS & 50 DAS & 60 DAS & 70 DAS & Average & 30 DAS & 40 DAS & 50 DAS & 60 DAS & 70 DAS & Average \\
\hline \multirow{2}{*}{$\mathrm{T}_{1}$} & 3.5 & 9.5 & 10.8 & 13.8 & 12.8 & 10.1 & 5.3 & 11.3 & 15.4 & 16.4 & 18.4 & 13.4 \\
& $(10.78) \mathrm{bc}$ & $(17.95)^{\mathrm{c}}$ & $(19.19) \mathrm{c}$ & $(21.81) \mathrm{b}$ & $(20.96) \mathrm{b}$ & $(18.53) \mathrm{d}$ & $(13.31) \mathrm{d}$ & $(19.64) \mathrm{bc}$ & $(23.11) \mathrm{b}$ & $(23.89) \mathrm{b}$ & $(25.40) \mathrm{b}$ & $(21.47) \mathrm{b}$ \\
\hline \multirow{2}{*}{$\mathrm{T}_{2}$} & 2.8 & 8.5 & 7.5 & 11.5 & 13.1 & 8.7 & 4.1 & 10.5 & 14.2 & 15.1 & 17.9 & 12.4 \\
& $(9.63) \mathrm{b}$ & $(16.95)^{\mathrm{b}}$ & $(15.89) \mathrm{b}$ & $(19.82) \mathrm{ab}$ & $(21.22) \mathrm{b}$ & $(17.15) \mathrm{cd}$ & $(11.68) \mathrm{c}$ & $(18.91) \mathrm{b}$ & $(22.14) \mathrm{b}$ & $(22.87) \mathrm{b}$ & $(25.03) \mathrm{b}$ & $(20.62) \mathrm{b}$ \\
\hline \multirow{2}{*}{$\mathrm{T}_{3}$} & 0 & 8.0 & 6.8 & 12.1 & 10.5 & 7.5 & 3.8 & 10.1 & 13.4 & 13.2 & 18.1 & 11.7 \\
& $(0.0) \mathrm{a}$ & $(16.43)^{\mathrm{b}}$ & $(15.12) \mathrm{b}$ & $(20.36) \mathrm{b}$ & $(18.91) \mathrm{b}$ & $(15.89) \mathrm{bc}$ & $(11.24) \mathrm{c}$ & $(18.53) \mathrm{b}$ & $(21.47) \mathrm{b}$ & $(21.30) \mathrm{b}$ & $(25.18) \mathrm{b}$ & $(20.00) \mathrm{b}$ \\
\hline \multirow{2}{*}{$\mathrm{T}_{4}$} & 0 & 4.3 & 3.5 & 8.5 & 5.8 & 4.4 & 1.7 & 5.3 & 6.8 & 8.9 & 10.8 & 6.7 \\
& $(0.0) \mathrm{a}$ & $(11.97) \mathrm{a}$ & $(10.78) \mathrm{a}$ & $(16.95) \mathrm{a}$ & $(13.94) \mathrm{a}$ & $(12.11) \mathrm{a}$ & $(7.49) \mathrm{a}$ & $(13.31) \mathrm{a}$ & $(15.12) \mathrm{a}$ & $(17.36) \mathrm{a}$ & $(19.19) \mathrm{a}$ & $(15.00) \mathrm{a}$ \\
\hline \multirow{2}{*}{$\mathrm{T}_{6}$} & 0 & 5.3 & 6.9 & 9.3 & 6.9 & 5.7 & 2.9 & 6.8 & 7.9 & 10.1 & 12.5 & 8.0 \\
& $(0.0) \mathrm{a}$ & $(13.31)^{\mathrm{a}} \mathrm{(15.23) \textrm {b }}$ & $(17.76) \mathrm{a}$ & $(15.23) \mathrm{a}$ & $(13.81) \mathrm{ab}$ & $(9.80) \mathrm{b}$ & $(15.12) \mathrm{a}$ & $(16.32) \mathrm{a}$ & $(18.53) \mathrm{a}$ & $(20.70) \mathrm{a}$ & $(16.43) \mathrm{a}$ \\
\hline \multirow{2}{*}{$\mathrm{T}_{7}$} & 4.2 & 10.5 & 12.4 & 15.1 & 14.5 & 11.3 & 9.3 & 15.2 & 18.4 & 20.5 & 22.5 & 17.2 \\
& $(11.83) \mathrm{c}$ & $(18.91)^{\mathrm{d}}$ & $(20.62) \mathrm{c}$ & $(22.87) \mathrm{b}$ & $(22.38) \mathrm{b}$ & $(19.64) \mathrm{d}$ & $(17.76) \mathrm{e}$ & $(22.95) \mathrm{c}$ & $(25.40) \mathrm{c}$ & $(26.92) \mathrm{c}$ & $(28.32) \mathrm{c}$ & $(24.50) \mathrm{c}$ \\
\hline $\mathrm{CD}$ & 1.21 & 4.01 & 2.54 & 3.11 & 3.57 & 2.89 & 1.15 & 2.89 & 3.17 & 1.47 & 2.09 & 2.15 \\
\hline $\mathrm{CV}$ & 13.9 & 11.5 & 16.4 & 9.7 & 13.4 & 12.9 & 18.5 & 11.2 & 13.4 & 14.5 & 10.8 & 13.7 \\
\hline
\end{tabular}

Mean with same letter are not significantly different at $5 \%$ level by Duncan's Multiple Range test

Values in parenthesis are arc sine transformed values; DAS: Days After Sowing

Table 8: Efficacy of chlorantraniliprole 625g/L FS against rice stem borer in terms of per cent dead hearts during rabi, 2019-2020

\begin{tabular}{|c|c|c|c|c|c|c|c|c|c|c|c|c|}
\hline & \multicolumn{5}{|c|}{ Rice variety } & \multicolumn{7}{|c|}{ Rice Hybrid } \\
\hline Treatments & 30 DAS & 40 DAS & 50 DAS & 60 DAS & 70 DAS & Average & 30 DAS & 40 DAS & 50 DAS & 60 DAS & 70 DAS & Average \\
\hline $\mathrm{T}_{1}$ & $\begin{array}{c}5.2 \\
(13.18) \mathrm{b}\end{array}$ & $\begin{array}{c}4.8 \\
(12.66) \mathrm{b}\end{array}$ & $\begin{array}{c}6.7 \\
(15.00) \mathrm{d}\end{array}$ & $\begin{array}{c}8.8 \\
(17.26) \mathrm{d}\end{array}$ & $\begin{array}{c}6.1 \\
(14.30) \mathrm{b}\end{array}$ & $\begin{array}{c}6.3 \\
(14.54) c\end{array}$ & $\begin{array}{c}6.3 \\
(14.54) b c\end{array}$ & $\begin{array}{c}5.1 \\
(13.05) b\end{array}$ & $\begin{array}{c}6.3 \\
(14.54) \mathrm{a}\end{array}$ & $\begin{array}{c}8.8 \\
(17.26) \mathrm{d}\end{array}$ & $\begin{array}{c}7.8 \\
(16.22) b\end{array}$ & $\begin{array}{c}6.9 \\
(15.23) b\end{array}$ \\
\hline $\mathrm{T}_{2}$ & $\begin{array}{c}4.8 \\
(12.66) \mathrm{b} \\
\end{array}$ & \begin{tabular}{|c|}
4.3 \\
$(11.97) \mathrm{b}$ \\
\end{tabular} & $\begin{array}{c}5.4 \\
(13.44) \mathrm{c} \\
\end{array}$ & $\begin{array}{c}8.1 \\
(16.54) \mathrm{d} \\
\end{array}$ & \begin{tabular}{|c|}
5.3 \\
$(13.31) \mathrm{b}$ \\
\end{tabular} & $\begin{array}{c}5.6 \\
(13.69) \mathrm{bc} \\
\end{array}$ & $\begin{array}{c}5.1 \\
(13.05) \mathrm{ab} \\
\end{array}$ & $\begin{array}{c}4.0 \\
(11.54) \mathrm{b} \\
\end{array}$ & $\begin{array}{c}5.5 \\
(13.56) \mathrm{a} \\
\end{array}$ & $\begin{array}{c}7.4 \\
(15.79) \mathrm{c} \\
\end{array}$ & $\begin{array}{c}6.5 \\
(14.77) \mathrm{ab} \\
\end{array}$ & $\begin{array}{c}5.7 \\
(13.81) \mathrm{a} \\
\end{array}$ \\
\hline $\mathrm{T}_{3}$ & $\begin{array}{c}5.1 \\
(13.05) b\end{array}$ & $\begin{array}{c}4.0 \\
(11.54) \mathrm{ab}\end{array}$ & $\begin{array}{c}5.1 \\
(13.05) \mathrm{c}\end{array}$ & $\begin{array}{c}7.3 \\
(15.68) \mathrm{cd}\end{array}$ & $\begin{array}{c}5.0 \\
(12.92) \mathrm{b}\end{array}$ & $\begin{array}{c}5.3 \\
(13.31) \mathrm{b}\end{array}$ & $\begin{array}{c}5.0 \\
(12.92) \mathrm{ab}\end{array}$ & $\begin{array}{c}3.9 \\
(11.39) \mathrm{ab}\end{array}$ & $\begin{array}{c}4.3 \\
(11.97) \mathrm{a}\end{array}$ & $\begin{array}{c}6.0 \\
(14.18) \mathrm{b} \\
\end{array}$ & $\begin{array}{c}6.3 \\
(14.54) \mathrm{ab}\end{array}$ & $\begin{array}{c}5.1 \\
(13.05) \mathrm{a}\end{array}$ \\
\hline $\mathrm{T}_{4}$ & \begin{tabular}{|c|}
2.2 \\
$(8.53) \mathrm{a}$ \\
\end{tabular} & \begin{tabular}{|c|}
1.8 \\
$(7.71) \mathrm{a}$ \\
\end{tabular} & $\begin{array}{c}3.2 \\
(10.30) \mathrm{ab} \\
\end{array}$ & $\begin{array}{c}4.3 \\
(11.97) \mathrm{ab} \\
\end{array}$ & \begin{tabular}{|c|}
2.1 \\
$(8.33) \mathrm{a}$ \\
\end{tabular} & $\begin{array}{c}2.7 \\
(9.46) \mathrm{a} \\
\end{array}$ & $\begin{array}{c}3.2 \\
(10.30) \mathrm{a} \\
\end{array}$ & \begin{tabular}{|c|}
2.8 \\
$(9.63) \mathrm{a}$ \\
\end{tabular} & \begin{tabular}{|c|}
3.8 \\
$(11.24) \mathrm{a}$ \\
\end{tabular} & $\begin{array}{c}4.1 \\
(11.68) \mathrm{a} \\
\end{array}$ & \begin{tabular}{|c|}
4.5 \\
$(12.25) \mathrm{a}$ \\
\end{tabular} & $\begin{array}{c}3.7 \\
(11.09) \mathrm{a} \\
\end{array}$ \\
\hline $\mathrm{T}_{6}$ & \begin{tabular}{|c|}
3.8 \\
$(11.24) \mathrm{b}$ \\
\end{tabular} & $\begin{array}{c}2.7 \\
(9.46) \mathrm{a} \\
\end{array}$ & $\begin{array}{c}4.0 \\
(11.54) \mathrm{bc} \\
\end{array}$ & $\begin{array}{c}5.6 \\
(13.69) \mathrm{bc} \\
\end{array}$ & $\begin{array}{c}3.8 \\
(11.24) \mathrm{ab} \\
\end{array}$ & $\begin{array}{c}4.0 \\
(11.54) \mathrm{ab} \\
\end{array}$ & $\begin{array}{c}4.3 \\
(11.97) \mathrm{a} \\
\end{array}$ & $\begin{array}{c}5.0 \\
(12.92) \mathrm{b} \\
\end{array}$ & \begin{tabular}{|c|}
4.8 \\
$(12.66) \mathrm{a}$ \\
\end{tabular} & $\begin{array}{c}5.3 \\
(13.31) \mathrm{b} \\
\end{array}$ & \begin{tabular}{|c|}
6.0 \\
$(14.18)$ \\
\end{tabular} & $\begin{array}{c}5.1 \\
(13.05) \mathrm{a} \\
\end{array}$ \\
\hline $\mathrm{T}_{7}$ & \begin{tabular}{|c|}
7.4 \\
$(15.79) \mathrm{c}$ \\
\end{tabular} & \begin{tabular}{|c|}
8.7 \\
$(17.15) b$ \\
\end{tabular} & $\begin{array}{c}10.5 \\
(18.91) \mathrm{e} \\
\end{array}$ & \begin{tabular}{|c|}
9.8 \\
$(18.24) \mathrm{e}$ \\
\end{tabular} & \begin{tabular}{|c|}
6.8 \\
$(15.12) \mathrm{b}$ \\
\end{tabular} & $\begin{array}{c}8.6 \\
(17.05) \mathrm{c} \\
\end{array}$ & $\begin{array}{c}8.7 \\
(17.15) \mathrm{c}\end{array}$ & $\begin{array}{c}11.2 \\
(19.55) \mathrm{c}\end{array}$ & $\begin{array}{c}13.8 \\
(21.81) \mathrm{b}\end{array}$ & $\begin{array}{c}10.9 \\
(19.28) \mathrm{e}\end{array}$ & \begin{tabular}{|c|}
8.5 \\
$(16.95) \mathrm{b}$ \\
\end{tabular} & $\begin{array}{c}10.6 \\
(19.00) b\end{array}$ \\
\hline $\mathrm{CD}$ & 2.28 & 3.85 & 1.53 & 2.04 & 4.53 & 2.85 & 2.91 & 4.93 & 3.63 & 1.04 & 3.47 & 3.12 \\
\hline $\mathrm{CV}$ & 18.54 & 12.71 & 14.7 & 13.5 & 11.8 & 14.3 & 14.3 & 10.4 & 9.7 & 12.5 & 10.4 & 11.5 \\
\hline
\end{tabular}

Mean with same letter are not significantly different at 5\% level by Duncan's Multiple Range test

Values in parenthesis are arc sine transformed values; DAS: Days After Sowing

\subsubsection{Influence of test chemical on incidence of rice leaf folder in terms of larval population}

The larval population of leaf folder ranged from 0.0 to 7.3 and 0.0 to 6.4 number per hill in rice variety and rice hybrid, respectively. Among various treatments, $\mathrm{T}_{4}$ (chlorantraniliprole 625 g/L FS @ 90 g a.i./ha) had recorded minimum per cent dead hearts at 30,40, 50, 60 and 70 DAS with $0.0,0.0,2.4,1.8 \& 3.3$ per cent in rice variety and with $0.0,0.0,0.0,1.9,2.8 \& 0.9$ per cent in rice hybrid, respectively. The decreasing order of efficacy in suppressing the average leaf folder population in rice variety and rice hybrid represents $\mathrm{T}_{4}(1.5 \& 0.9)>\mathrm{T}_{6}(2.0 \& 1.7)>\mathrm{T}_{3}(3.2 \&$ 2.4) $>\mathrm{T}_{2}(3.6 \& 2.6)>\mathrm{T}_{1}(4.0 \& 2.8)>\mathrm{T}_{7}(4.7 \& 3.8)$ (Table 9$)$.

\subsubsection{Influence of test chemical on incidence of rice leaf folder in terms of per cent damage}

The leaf folder incidence in terms percent damage leaves also represented the similar trend in case with various treatments imposed. At 30 DAS, the damage per cent was nil both in case with rice hybrid and rice variety. At 40 DAS the damage was nil in $T_{4}$ and $T_{6}$ in rice variety whereas, at 40 and 50 DAS the damage was nil in $T_{3}, T_{4}$ and $T_{6}$ representing efficacy in reducing the pest incidence.

The average per cent damaged leaves in rice variety recorded to be lowest in $\mathrm{T}_{4}(3.0 \& 1.9)$ and found on par to $\mathrm{T}_{6}(3.6 \&$ 2.8) followed by least damage recorded in $\mathrm{T}_{3}(5.5 \& 3.7), \mathrm{T}_{2}$ $(6.0 \& 4.1), \mathrm{T}_{1}(7.0 \& 4.8)$ and highest damage recorded in $\mathrm{T}_{7}$ $(8.1 \& 6.7)$ with respect to rice varierty and rice hybrid, respectively (Table 10).

\subsection{Efficacy of test chemicals (cumulative mean) in terms of per cent reduction over control}

The pooled mean in terms of per cent reduction over control of stem borer and leaf folder in both rice variety and rice hybrid was calculated for both the seasons.

3.4.1 Rice stem borer: During the season kharif, 2019 the order of efficacy of various chemicals with respect to per cent dead hearts represents $\mathrm{T}_{4}(76.71 \& 78.19)>\mathrm{T}_{6}(58.44 \&$ 73.68) $>\mathrm{T}_{3}(44.74 \& 54.51)>\mathrm{T}_{2}(46.12 \& 50.37)>\mathrm{T}_{1}(35.61$ $\& 42.48)$ in rice variety and rice hybrid, respectively. During rabi, 2019-2020 similar trend was observed as in case with 
kharif 2019 and among various treatments the highest per cent reduction was recorded in $\mathrm{T} 4$ followed by $\mathrm{T} 6, \mathrm{~T} 3, \mathrm{~T} 2$ and $\mathrm{T} 1$ with $68.60,53.48,38.37,34.88 \& 26.74$ and $65.09,51.88$,
$51.88,46.22 \& 33.94$ per cent in rice variety and rice hybrid, respectively (Table 11).

Table 9: Efficacy of chlorantraniliprole 625g/L FS on larval population of rice leaf folder during rabi, 2019-2020

\begin{tabular}{|c|c|c|c|c|c|c|c|c|c|c|c|c|c|}
\hline & \multicolumn{9}{|c|}{ Rice variety } & \multicolumn{9}{c|}{ Rice Hybrid } \\
\hline Treatments & 30 DAS & 40 DAS & 50 DAS & 60 DAS & 70 DAS & Average & 30 DAS & 40 DAS & 50 DAS & 60 DAS & 70 DAS & Average \\
\hline \multirow{2}{*}{$\mathrm{T}_{1}$} & 0 & 4.1 & 5.4 & 3.8 & 6.5 & 4.0 & 0 & 0 & 3.5 & 4.8 & 5.9 & 2.8 \\
& $(0.0)$ & $(2.02) \mathrm{bc}$ & $(2.32) \mathrm{ab}$ & $(1.95) \mathrm{cd}$ & $(2.55) \mathrm{b}$ & $(2.00)$ & $(0.0)$ & $(0.0)$ & $(1.87) \mathrm{b}$ & $(2.19) \mathrm{c}$ & $(2.43) \mathrm{cd}$ & $(1.67) \mathrm{c}$ \\
\hline \multirow{2}{*}{$\mathrm{T}_{2}$} & 0 & 3.7 & 5.0 & 3.5 & 6.0 & 3.6 & 0 & 0 & 3.2 & 4.3 & 5.3 & 2.6 \\
& $(0.0)$ & $(1.92) \mathrm{a}$ & $(2.24) \mathrm{ab}$ & $(1.87) \mathrm{c}$ & $(2.45) \mathrm{b}$ & $(1.90)$ & $(0.0)$ & $(0.0)$ & $(1.79) \mathrm{ab}$ & $(2.07) \mathrm{bc}$ & $(2.30) \mathrm{cd}$ & $(1.61) \mathrm{bc}$ \\
\hline \multirow{2}{*}{$\mathrm{T}_{3}$} & 0 & 2.8 & 4.9 & 3.0 & 5.4 & 3.2 & 0 & 0 & 2.8 & 4.0 & 5.0 & 2.4 \\
& $(0.0)$ & $(1.67) \mathrm{a}$ & $(2.21) \mathrm{a}$ & $(1.73) \mathrm{bc}$ & $(2.32) \mathrm{b}$ & $(1.79)$ & $(0.0)$ & $(0.0)$ & $(1.67) \mathrm{a}$ & $(2.00) \mathrm{b}$ & $(2.24) \mathrm{c}$ & $(1.55) \mathrm{b}$ \\
\hline \multirow{2}{*}{$\mathrm{T}_{4}$} & 0 & 0 & 2.4 & 1.8 & 3.3 & 1.5 & 0 & 0 & 0 & 1.9 & 2.8 & 0.9 \\
& $(0.0)$ & $(0.0)$ & $(1.55) \mathrm{a}$ & $(1.34) \mathrm{a}$ & $(1.82) \mathrm{a}$ & $(1.22)$ & $(0.0)$ & $(0.0)$ & $(0.0) \mathrm{a}$ & $(1.38) \mathrm{a}$ & $(1.67) \mathrm{ab}$ & $(0.95) \mathrm{a}$ \\
\hline \multirow{2}{*}{$\mathrm{T}_{6}$} & 0 & 0 & 3.1 & 2.2 & 4.5 & 2.0 & 0 & 0 & 2.4 & 2.8 & 3.4 & 1.7 \\
& $(0.0)$ & $(0.0)$ & $(1.76) \mathrm{a}$ & $(1.48) \mathrm{ab}$ & $(2.12) \mathrm{ab}$ & $(1.41)$ & $(0.0)$ & $(0.0)$ & $(1.55) \mathrm{a}$ & $(1.67) \mathrm{ab}$ & $(1.84) \mathrm{b}$ & $(1.30) \mathrm{b}$ \\
\hline \multirow{2}{*}{$\mathrm{T}_{7}$} & 0 & 5.2 & 6.5 & 4.3 & 7.3 & 4.7 & 0 & 3.5 & 3.9 & 5.3 & 6.4 & 3.8 \\
& $(0.0)$ & $(2.28) \mathrm{c}$ & $(2.55) \mathrm{b}$ & $(2.07) \mathrm{d}$ & $(2.70) \mathrm{b}$ & $(2.17)$ & $(0.0)$ & $(1.87)$ & $(1.97) \mathrm{b}$ & $(2.30) \mathrm{c}$ & $(2.53) \mathrm{d}$ & $(1.95) \mathrm{c}$ \\
\hline $\mathrm{CD}$ & $\mathrm{NS}$ & 0.44 & 0.84 & 0.31 & 0.61 & 0.55 & $\mathrm{NS}$ & $\mathrm{NS}$ & 0.24 & 0.47 & 0.37 & 0.36 \\
\hline $\mathrm{CV}$ & - & 12.4 & 18.4 & 13.71 & 15.4 & 14.97 & - & - & 12.5 & 13.8 & 9.7 & 12.0 \\
\hline
\end{tabular}

Mean with same letter are not significantly different at 5\% level by Duncan's Multiple Range test

Values in parenthesis are square root transformed values; DAS: Days After Sowing

Table 10: Efficacy of chlorantraniliprole 625g/L FS against rice leaf folder in terms of per cent damage during rabi, 2019-2020

\begin{tabular}{|c|c|c|c|c|c|c|c|c|c|c|c|c|}
\hline & \multicolumn{9}{|c|}{ Rice variety } & \multicolumn{1}{c|}{ Rice Hybrid } \\
\hline Treatments 30 DAS 40 DAS & 50 DAS & 60 DAS & 70 DAS & Average 30 DAS & 40 DAS & 50 DAS & 60 DAS & 70 DAS & Average \\
\hline \multirow{2}{*}{$\mathrm{T}_{1}$} & 0 & 2.1 & 8.8 & 10.2 & 13.8 & 7.0 & 0 & 0 & 4.8 & 8.8 & 10.4 & 4.8 \\
& $(0.0)$ & $(8.33) \mathrm{b}$ & $(17.26) \mathrm{b} \mathrm{c}$ & $(18.63) \mathrm{bc}$ & $(21.81) \mathrm{c}$ & $(15.34)$ & $(0.0)$ & $(0.0) \mathrm{a}$ & $(12.66) \mathrm{bc}$ & $(17.26) \mathrm{bc}$ & $(18.81) \mathrm{b}$ & $(12.66) \mathrm{bc}$ \\
\hline \multirow{2}{*}{$\mathrm{T}_{2}$} & 0 & 1.5 & 7.2 & 9.8 & 11.5 & 6.0 & 0 & 0 & 4.1 & 7.4 & 9.1 & 4.1 \\
& $(0.0)$ & $(7.03) \mathrm{b}$ & $(15.56) \mathrm{b}$ & $(18.24) \mathrm{b}$ & $(19.82) \mathrm{b}$ & $(14.18)$ & $(0.0)$ & $(0.0) \mathrm{a}$ & $(11.68) \mathrm{bc}$ & $(15.79) \mathrm{ab}$ & $(17.56) \mathrm{b}$ & $(11.68) \mathrm{b}$ \\
\hline \multirow{2}{*}{$\mathrm{T}_{3}$} & 0 & 1.5 & 6.8 & 8.5 & 10.8 & 5.5 & 0 & 0 & 3.5 & 6.3 & 8.5 & 3.7 \\
& $(0.0)$ & $(7.03) \mathrm{b}$ & $(15.12) \mathrm{b}$ & $(16.95) \mathrm{b}$ & $(19.19) \mathrm{b}$ & $(13.56)$ & $(0.0)$ & $(0.0) \mathrm{a}$ & $(10.78) \mathrm{bc}$ & $(14.54) \mathrm{ab}$ & $(16.95) \mathrm{b}$ & $(11.09) \mathrm{b}$ \\
\hline \multirow{2}{*}{$\mathrm{T}_{4}$} & 0 & 0 & 3.5 & 4.3 & 7.1 & 3.0 & 0 & 0 & 0 & 4.3 & 5.0 & 1.9 \\
& $(0.0)$ & $(0.0) \mathrm{a}$ & $(10.78) \mathrm{a}$ & $(11.97) \mathrm{a}$ & $(15.45) \mathrm{a}$ & $(9.97) \mathrm{a}$ & $(0.0)$ & $(0.0) \mathrm{a}$ & $(0.0) \mathrm{a}$ & $(11.97) \mathrm{a}$ & $(12.92) \mathrm{a}$ & $(7.92) \mathrm{a}$ \\
\hline \multirow{2}{*}{$\mathrm{T}_{6}$} & 0 & 0 & 4.3 & 5.7 & 7.9 & 3.6 & 0 & 0 & 2.4 & 5.4 & 6.3 & 2.8 \\
& $(0.0)$ & $(0.0) \mathrm{a}$ & $(11.97) \mathrm{a}$ & $(13.81) \mathrm{a}$ & $(16.32) \mathrm{a}$ & $(10.94) \mathrm{a}$ & $(0.0)$ & $(0.0) \mathrm{a}$ & $(8.91) \mathrm{b}$ & $(13.44) \mathrm{ab}$ & $(14.54) \mathrm{a}$ & $(9.63) \mathrm{ab}$ \\
\hline \multirow{2}{*}{$\mathrm{T} 7$} & 0 & 3.3 & 10.5 & 12.4 & 14.5 & 8.1 & 0 & 3.4 & 5.9 & 10.4 & 13.8 & 6.7 \\
& $(0.0)$ & $(10.47) \mathrm{c}$ & $(18.91) \mathrm{c}$ & $(20.62) \mathrm{c}$ & $(22.38) \mathrm{d}$ & $(16.54)$ & $(0.0)$ & $(10.63) \mathrm{b}$ & $(14.06) \mathrm{c}$ & $(18.81) \mathrm{c}$ & $(21.81) \mathrm{c}$ & $(15.00) \mathrm{c}$ \\
\hline $\mathrm{CD}$ & $\mathrm{NS}$ & 1.34 & 2.84 & 2.07 & 1.87 & 2.03 & $\mathrm{NS}$ & 3.54 & 4.54 & 3.97 & 2.14 & 3.55 \\
\hline $\mathrm{CV}$ & - & 18.5 & 11.8 & 9.8 & 15.4 & 13.9 & - & 12.9 & 17.45 & 9.54 & 11.44 & 10.69 \\
\hline
\end{tabular}

Mean with same letter are not significantly different at 5\% level by Duncan's Multiple Range test

Values in parenthesis are arc sine transformed values; DAS: Days After Sowing

Table 11: Efficacy of chlorantraniliprole $625 \mathrm{~g} / \mathrm{L} \mathrm{FS}$ on incidence of rice stem borer in terms of per cent reduction over control

\begin{tabular}{|c|c|c|c|c|c|c|c|c|}
\hline \multirow{2}{*}{ Treatments } & \multicolumn{4}{|c|}{ Kharif, 2019 } & \multicolumn{4}{c|}{ rabi, 2019-2020 } \\
\cline { 2 - 9 } & \multicolumn{2}{|c|}{ Rice variety } & \multicolumn{2}{c|}{ Rice hybrid } & \multicolumn{2}{c|}{ Rice variety } & \multicolumn{2}{c|}{ Rice hybrid } \\
\hline & \%DH & \% ROC & Per cent DH & \% ROC & \% DH & \% ROC & \%DH & \% ROC \\
\hline $\mathrm{T}_{1}$ & $\begin{array}{c}14.1 \\
(22.06)^{\mathrm{b}}\end{array}$ & 35.61 & $\begin{array}{c}15.3 \\
(23.03)^{\mathrm{b}}\end{array}$ & 42.48 & $\begin{array}{c}6.3 \\
(14.54) \mathrm{c}\end{array}$ & 26.74 & $\begin{array}{c}6.9 \\
(15.23) \mathrm{b}\end{array}$ & 33.94 \\
\hline $\mathrm{T}_{2}$ & $\begin{array}{c}11.8 \\
(20.09)^{\mathrm{b}}\end{array}$ & 46.12 & $\begin{array}{c}13.2 \\
(21.30)^{\mathrm{b}}\end{array}$ & 50.37 & $\begin{array}{c}5.6 \\
(13.69) \mathrm{bc}\end{array}$ & 34.88 & $\begin{array}{c}5.7 \\
(13.81) \mathrm{a}\end{array}$ & 46.22 \\
\hline $\mathrm{T}_{3}$ & $\begin{array}{c}12.1 \\
(20.36)^{\mathrm{b}}\end{array}$ & 44.74 & $\begin{array}{c}12.1 \\
(20.36)^{\mathrm{b}}\end{array}$ & 54.51 & $\begin{array}{c}5.3 \\
(13.31) \mathrm{b}\end{array}$ & 38.37 & $\begin{array}{c}5.1 \\
(13.05) \mathrm{a}\end{array}$ & 51.88 \\
\hline $\mathrm{T}_{4}$ & $\begin{array}{c}5.1 \\
(13.05)^{\mathrm{a}}\end{array}$ & 76.71 & $\begin{array}{c}5.8 \\
(13.94)^{\mathrm{a}}\end{array}$ & 78.19 & $\begin{array}{c}2.7 \\
(9.46) \mathrm{a}\end{array}$ & 68.60 & $\begin{array}{c}3.7 \\
(11.09) \mathrm{a}\end{array}$ & 65.09 \\
\hline $\mathrm{T}_{6}$ & $\begin{array}{c}9.1 \\
(17.56)^{\mathrm{b}}\end{array}$ & 58.44 & $\begin{array}{c}7.0 \\
(15.34)^{\mathrm{a}}\end{array}$ & 73.68 & $\begin{array}{c}4.0 \\
(11.54) \mathrm{ab}\end{array}$ & 53.48 & $\begin{array}{c}5.1 \\
(13.05) \mathrm{a}\end{array}$ & 51.88 \\
\hline $\mathrm{T}_{7}$ & $\begin{array}{c}21.9 \\
(27.90)^{\mathrm{c}}\end{array}$ & - & $\begin{array}{c}26.6 \\
(31.05)^{\mathrm{c}}\end{array}$ & - & $\begin{array}{c}8.6 \\
(17.05) \mathrm{c}\end{array}$ & - & $\begin{array}{c}10.6 \\
(19.00) \mathrm{b}\end{array}$ & - \\
\hline $\mathrm{CD}$ & 5.15 & & 2.68 & & 2.85 & & 3.12 & \\
\hline $\mathrm{CV}$ & 13.54 & & 10.15 & & 14.3 & & 11.5 & \\
\hline
\end{tabular}

3.4.2 Leaf folder in terms of damaged leaves: The highest per cent reduction of leaf folder larval population over control during kharif, 2019 was recorded in $\mathrm{T}_{4}$ treated plots (61.06) followed by $\mathrm{T}_{6}, \mathrm{~T}_{3}, \mathrm{~T}_{2}$ and $\mathrm{T}_{1}$ with 49.55, 33.62, 23.00 and 10.61 per cent in rice variety. The corresponding values in case with rice hybrid represents $\mathrm{T}_{4}(61.04)>\mathrm{T}_{6}(53.48)>\mathrm{T}_{3}$ (31.97) $>\mathrm{T}_{2}$ (27.91) $>\mathrm{T}_{1}$ (22.09). For the season rabi, 20192020 similar trend was recorded and the decreasing order of efficacy in suppressing the larval population represents $\mathrm{T}_{4}$ $(62.96 \& 71.64)>\mathrm{T}_{6}(55.55 \& 58.21)>\mathrm{T}_{3}(32.09 \& 44.77)$ 
$>\mathrm{T}_{2}(25.92 \& 38.88)>\mathrm{T}_{1}(13.58 \& 28.35)$ in rice variety and rice hybrid, respectively (Table 12).

\subsection{Influence of various treatments on natural enemies population}

The population of predatory spiders and mirid bugs were recorded from each plot and the mean population inferred that there is no significant difference among various treatments and all are found on par to each. It was apparent from the results that, the application of test chemical chlorantraniliprole $625 \mathrm{~g} / \mathrm{L}$ FS at various doses did not exterted any adverse effects to natural enemies at both kharif and rabi seasons under study period of 2019-2020.

\section{Phytotoxicity}

The results revealed that chlorantraniliprole 625 g/L FS @ 90 and $180 \mathrm{~g}$ a.i ha ${ }^{-1}$ have not exhibited any phytotoxic symptoms like leaf injury on tips and leaf surface, wilting, leaf vein clearing, necrosis, epinasty and hyponasty in any of the doses and treatments tested in rice crop. hlorantraniliprole $625 \mathrm{~g} / \mathrm{L}$ FS was tested for phytotoxicity at all the doses during kharif, 2019 and rabi, 2019-2020 and was also proven safe to rice crop during entire crop season.

\section{Efficacy of various treatments on yield}

The yield from various treated plots was recorded and converted in to $\mathrm{Kg} / \mathrm{ha}$. Among various treatments evaluated the highest yield was recorded in $\mathrm{T}_{4}$ and $\mathrm{T}_{6}$ with $4580 \& 4490$ and $4925 \& 4800 \mathrm{~kg} / \mathrm{ha}$ as against lowest yield with 2750 \& 2950 in untreated control during kharif, 2019 season with respect to rice variety and rice hybrid, respectively. During rabi, 2019-2020 the highest yields were realized in plots treated with $\mathrm{T}_{4}$ and $\mathrm{T}_{6}$ in both rice variety and rice hybrid with $3420 \& 3385$ and $3250 \& 3150 \mathrm{~kg} / \mathrm{ha}$, respectively (Table 13). This is for kind intimation that the yield was low in rabi, 2019-2020 due to lockdown effect (insufficient laborer).

Table 12: Efficacy of chlorantraniliprole $625 \mathrm{~g} / \mathrm{L} \mathrm{FS}$ on incidence of rice leaf folder in terms of per cent reduction over control

\begin{tabular}{|c|c|c|c|c|c|c|c|c|}
\hline \multirow{3}{*}{ Treatments } & \multicolumn{4}{|c|}{ Kharif, 2019} & \multicolumn{4}{|c|}{ rabi, 2019-2020 } \\
\hline & \multicolumn{2}{|c|}{ Rice variety } & \multicolumn{2}{|c|}{ Rice hybrid } & \multicolumn{2}{|c|}{ Rice variety } & \multicolumn{2}{|c|}{$\begin{array}{l}\text { Rice hybrid } \\
\end{array}$} \\
\hline & \%LFDL & $\%$ ROC & \%LFDL & $\%$ ROC & \%LFDL & $\%$ ROC & \%LFDL & $\%$ ROC \\
\hline $\mathrm{T}_{1}$ & $\begin{array}{c}10.1 \\
(18.53) d\end{array}$ & 10.61 & $\begin{array}{c}13.4 \\
(21.47) b\end{array}$ & 22.09 & $\begin{array}{c}7.0 \\
(15.34) \\
\end{array}$ & 13.58 & $\begin{array}{c}4.8 \\
(12.66) \mathrm{bc}\end{array}$ & 28.35 \\
\hline $\mathrm{T}_{2}$ & $\begin{array}{c}8.7 \\
(17.15) \mathrm{cd} \\
\end{array}$ & 23.00 & $\begin{array}{c}12.4 \\
(20.62) \mathrm{b}\end{array}$ & 27.91 & $\begin{array}{c}6.0 \\
(14.18) \\
\end{array}$ & 25.92 & $\begin{array}{c}4.1 \\
(11.68) \mathrm{b}\end{array}$ & 38.88 \\
\hline $\mathrm{T}_{3}$ & $\begin{array}{c}7.5 \\
(15.89) \mathrm{bc}\end{array}$ & 33.62 & $\begin{array}{c}11.7 \\
(20.00) b\end{array}$ & 31.97 & $\begin{array}{c}5.5 \\
(13.56) \\
\end{array}$ & 32.09 & $\begin{array}{c}3.7 \\
(11.09) \mathrm{b}\end{array}$ & 44.77 \\
\hline $\mathrm{T}_{4}$ & $\begin{array}{c}4.4 \\
(12.11) \mathrm{a} \\
\end{array}$ & 61.06 & $\begin{array}{c}6.7 \\
(15.00) \mathrm{a} \\
\end{array}$ & 61.04 & $\begin{array}{c}3.0 \\
(9.97)\end{array}$ & 62.96 & $\begin{array}{c}1.9 \\
(7.92) \mathrm{a} \\
\end{array}$ & 71.64 \\
\hline $\mathrm{T}_{6}$ & $\begin{array}{c}5.7 \\
(13.81) \mathrm{ab}\end{array}$ & 49.55 & $\begin{array}{c}8.0 \\
(16.43) \mathrm{a} \\
\end{array}$ & 53.48 & $\begin{array}{c}3.6 \\
(10.94) \\
\end{array}$ & 55.55 & $\begin{array}{c}2.8 \\
(9.63) \mathrm{ab}\end{array}$ & 58.21 \\
\hline $\mathrm{T}_{7}$ & $\begin{array}{c}11.3 \\
(19.64) d\end{array}$ & - & $\begin{array}{c}17.2 \\
(24.50) \mathrm{c}\end{array}$ & - & $\begin{array}{c}8.1 \\
(16.54)\end{array}$ & - & $\begin{array}{c}6.7 \\
(15.00) \mathrm{c}\end{array}$ & - \\
\hline $\mathrm{CD}$ & 2.89 & & 2.15 & & 2.03 & & 3.55 & \\
\hline $\mathrm{CV}$ & 12.9 & & 13.7 & & 13.9 & & 10.69 & \\
\hline
\end{tabular}

Mean with same letter are not significantly different at 5\% level by Duncan's Multiple Range test

values in parenthesis are arc sine transformations;\%LFDL: per cent leaf folder damaged leaves;\%ROC: Per cent reduction over control

Table 13: Efficacy of chlorantraniliprole $625 \mathrm{~g} / \mathrm{L} \mathrm{FS}$ on rice yield $(\mathrm{kg} / \mathrm{ha})$

\begin{tabular}{|c|c|c|c|c|c|c|c|c|}
\hline Treatments & \multicolumn{3}{|c|}{ kharif, 2019 } & \multicolumn{4}{c|}{ rabi, 2019-2020 } \\
\hline & \multicolumn{2}{|c|}{ Rice variety } & \multicolumn{2}{c|}{ Rice hybrid } & \multicolumn{2}{c|}{ Rice variety } & \multicolumn{2}{c|}{ Rice hybrid } \\
\hline & $\begin{array}{c}\text { Yield } \\
\text { (kg/ha) }\end{array}$ & $\begin{array}{c}\text { Yield advantage } \\
\text { over control }\end{array}$ & $\begin{array}{c}\text { Yield } \\
(\mathbf{k g} / \mathbf{h a})\end{array}$ & $\begin{array}{c}\text { Yield advantage } \\
\text { over control }\end{array}$ & $\begin{array}{c}\text { Yield } \\
(\mathbf{k g} / \mathbf{h a})\end{array}$ & $\begin{array}{c}\text { Yield advantage } \\
\text { over control }\end{array}$ & $\begin{array}{c}\text { Yield } \\
\text { (kg/ha) }\end{array}$ & $\begin{array}{c}\text { Yield advantage } \\
\text { over control }\end{array}$ \\
\hline $\mathrm{T}_{1}$ & $3950 \mathrm{c}$ & 43.6 & $4018 \mathrm{c}$ & 36.2 & $3050 \mathrm{~d}$ & 41.86 & $2955 \mathrm{~b}$ & 47.7 \\
\hline $\mathrm{T}_{2}$ & $3940 \mathrm{c}$ & 43.2 & $4010 \mathrm{c}$ & 35.9 & $3040 \mathrm{~d}$ & 41.39 & $2930 \mathrm{~b}$ & 46.5 \\
\hline $\mathrm{T}_{3}$ & $4080 \mathrm{~b}$ & 48.3 & $4210 \mathrm{~b}$ & 42.7 & $3205 \mathrm{c}$ & 49.06 & $3050 \mathrm{~b}$ & 52.5 \\
\hline $\mathrm{T}_{4}$ & $4580 \mathrm{a}$ & 66.5 & $4925 \mathrm{a}$ & 66.9 & $3420 \mathrm{a}$ & 59.06 & $3250 \mathrm{a}$ & 62.5 \\
\hline $\mathrm{T}_{6}$ & $4490 \mathrm{a}$ & 63.3 & $4800 \mathrm{a}$ & 62.7 & $3385 \mathrm{~b}$ & 57.44 & $3150 \mathrm{a}$ & 57.5 \\
\hline $\mathrm{T}_{7}$ & $2750 \mathrm{~d}$ & - & $2950 \mathrm{~d}$ & - & $2150 \mathrm{e}$ & - & $2000 \mathrm{c}$ & - \\
\hline $\mathrm{CD}$ & 88.7 & & 78.2 & & 110.4 & & 124.8 & \\
\hline $\mathrm{CV}$ & 14.7 & & & & & & & \\
\hline
\end{tabular}

Mean with same letter are not significantly different at 5\% level by Duncan's Multiple Range test

The research trials by Sachan et al. (2018) ${ }^{[8]}$ pronounced that among various insecticides application of chlorantraniliprole 18.5 SC @ 150ml/ha and chlorantraniliprole $0.4 \% \mathrm{G} @ 10$ $\mathrm{Kg} / \mathrm{ha}$ had registered significant efficacy in reducing stem borer incidence in basmai rice. Similarly the studies by Arvind and Rajpoot (2017) [9] inferred that application of Coragen 20\%@150ml/ha was most effective in reducing per cent dead hearts (4.15-4.65) and per cent white ears(5.70$6.25)$ as against highest in case with untreated control with 15.05-16.25 and 25.30-28.65 per cent dead hearts and white ears, respectively.

\section{Conclusions}

Among the various treatments evaluated for efficacy against stem borer and leaf folder infesting rice as seed dresser the treatment $\mathrm{T}_{4}$ (chlorantraniliprole $625 \mathrm{~g} / \mathrm{L} \mathrm{FS} @ 90 \mathrm{~g}$ a.i. /ha) had recorded significantly lowest per cent dead hearts, minimum leaf folder larval population and least per cent leaf folder damaged leaves in both rice variety and rice hybrid during kharif, 2019 and rabi, 2019-2020. The treatment $\mathrm{T}_{4}$ was found on par to $T_{6}$ in many cases in suppressing the stem borer and leaf folder damage. From the results, it is inferred that, chlorantraniliprole 625 g/L FS @ $90 \mathrm{~g}$ a.i. /ha (lumivia) was effective in suppressing the stemborer and leaf folder 
infestation up to 70 DAS if used as seed dresser in direct seeded rice with no harm to natural enemies and no phytotoxicity on rice crop.

\section{References}

1. Kakde AM, Patel KG. Seasonal incidence of rice yellow stem borer (Scirpophaga incertulas Wlk.) in relation to conventional and Sri Methods of planting and its correlation with weather parameters. J. Agric and Vet Science. 2014; 7(6):5-10.

2. www. Indiastat.com. Ministry of Agriculture. 2014-15. Government of India. http://www. Indiastat.com

3. Behura N, Sen P, Kar MK. Introgression of yellow stem borer (Scirpophaga incertulas) resistance gene, into cultivated rice (Oryza sp.) from wild spp. Indian Journal Agriculture Science. 2011; 81:359-62.

4. Yarasi B, Sadumpati V, Immanni CP, Vudem DR, Khareedu VR. Transgenic rice expressing Allium sativum leaf agglutinin (ASAL) exhibits highlevel resistance against major sap-sucking pests. BMC Plant Biology. 2008; 8:102-115.

5. Deka S, Barthakur S. Overview on current status of biotechnological interventions on yellow stem borer, Scirpophaga incertulas (Lepidoptera: Crambidae) resistance in rice. Biotechnol Adv. 2010; 28(1):70-81.

6. Teng PS, Heong KL, Keith M. Advances in tropical rice pest management research. In: New frontiers in rice research (Eds.: K. Muralidharan and E. A. Siddiq).Directorate of Rice Research, Hyderabad, India, 1993, 255

7. Ramasamy $\mathrm{C}$, Jaliecksono $\mathrm{T}$. Inter country comparison of insect and disease losses. In: Rice Research in Asia: Progress and Priorities. (Eds.: R.E.R.W. Evanson, W. Herdt and M. Hossain), CAB International and IRRI, Philippines, 1996, 26.

8. Sachan SK, Kashyap AK, Ritesh Sharma, Verma KD, Singh HR. Efficacy of some novel insecticides against yellow stem borer, Scirpophaga incertulas (Walker) In Basmati Rice. Journal of Pharmacognosy and Phytochemistry 2018; SP1:195-197.

9. Arvind KS, Rajpoot SKS. Efficacy of commercially available insecticides and botanicals against yellow stem borer (Scirpophaga incertulas, Walker) of rice. Bulletin of Environment, Pharmacology and Life Sciences. 2017; $6(3): 11-13$ 